\title{
One-Dimensional Lattice Gas Models with Infinitely Many Absorbing States
}

\author{
Carlos E. Fiore and Mário J. de Oliveira \\ Instituto de Física \\ Universidade de São Paulo \\ Caixa Postal 66318 \\ 05315-970 São Paulo, São Paulo, Brazil
}

Received on 6 December, 2005

\begin{abstract}
We determined, by numerical simulations, the critical behavior one-dimensional systems with infinitely many absorbing states. These models are modified versions of the conserved lattice gas model in which two adjacent active particles instead of just one can jump to their neighboring sites. The models studied here are examples of systems with many absorbing states and order parameter coupled to a nondiffusive conserved field that show nontrivial critical behavior.
\end{abstract}

Keywords: Self-organized criticality; Absorbing phase transitions; Many absorbing states

\section{INTRODUCTION}

One of the major tasks in the study of nonequilibrium phase transitions is to determine the basic ingredients that distinguish the different universality classes. Nonequilibrium phase transitions to a unique absorbing state generically belong to the universality class of directed percolation. This conjecture has been proposed by Grassberger [1] and later extended by Grinstein et al [2]. It establishes that systems with a scalar order parameter exhibiting a continuous phase transition to a unique absorbing state generically belong to the universality class of directed percolation. Directed Percolation defines a precise universality class (described by the Reggeon field theory) which is very robust with respect to the introduction of microscopic modifications.

Subsequently Rossi at al [3] have proposed a new universality class for the critical behavior of systems that possess infinitely many absorbing states and a nondiffusive conserved field. Several models have been studied to check this new finding, as the fixed-energy sandpile (FES) models [4-6], the conservative lattice gas (CLG) model $[3,7,8]$, the conserved transfer threshold process (CTTP) $[3,9]$ and reactiondiffusion systems [10]. More recently, the Langevin equation describing systems in which the order parameter is coupled to a conserved nondiffusive field [11] has been solved numerically and the critical exponents have been determined [12,13].

With the exception of the CLG, all models mentioned above present a nontrivial critical behavior in one dimension. The CLG model defined on a one-dimensional chain can be solved exactly [14] and shows a classical critical behavior. For instance, the order parameter vanishes linearly when half of the sites are occupied. In this paper, we consider three modified versions of the CLG model, two of them exhibiting nonclassical critical behavior, which is compatible with calculation from a Langevin equation $[12,13]$. Whereas in the ordinary CLG models just one active particle jumps to a nearby empty site, in the modified versions two active particles jump to nearby empty sites. This process, that produces two-site instead of just one-site vacancies, together with the conservation of particles enhances the creation of active sites specially when the density of particles is less then but near 0.5 and may give rise to an active state for a density strictly less than 0.5 .

\section{MODELS}

The models we have studied are defined on a chain of $L$ sites with periodic boundary conditions. A configuration is specified by the occupation variable $\eta_{i}$ that assumes the value $\eta_{i}=0$ if the site $i$ is empty and $\eta_{i}=1$ if the site $i$ is occupied by a particle. Double or multiple occupancies are forbidden. Isolated particles (particles with empty next-nearest-neighbor sites) are inactive and do not move. Adjacent sites occupied by particles are active and repel each other via repulsive shortrange interactions. As a product of this interaction, the two active particles may jump to their neighborhoods. The continuous time Markovian dynamics consists of a series of repulsions events in active states.

Differently from the dynamics of the CLG model, in which just one particle jumps at each time step, in the present models both adjacent active particles may jump to their neighboring sites. Three versions are studied that are called model 1, 2 and 3 , which differ by their neighborhood. In model 1 the neighborhood is composed by the first-neighbor site only. In model 2 , by the first and second neighboring sites, and in model 3 , by the first, second and third neighboring sites. In addition, the neighborhood of the right (left) particle is at its right (left). If there are $n$ empty site in the neighborhood, the jumping probability to an empty site equals $1 / n$. If all sites of the neighborhood are occupied the particle remains at its place. In model 2 , we have also considered a simpler model that consists in forbidding jumps to the second nearest-neighbor site when $n=1$. Thus, in this model, for $n=1$, active sites can only jump to their first nearest-neighbor site.

In all models studied here, the number of particles is conserved and the density of particles $\rho$ works as the control parameter. In the regime of small densities, the system falls in one of its many absorbing states (without repulsions). Increasing the density of particles, a phase transition takes place to the active state (with repulsions) at the critical density $\rho_{c}$. The order parameter of the system is the density of active particles 


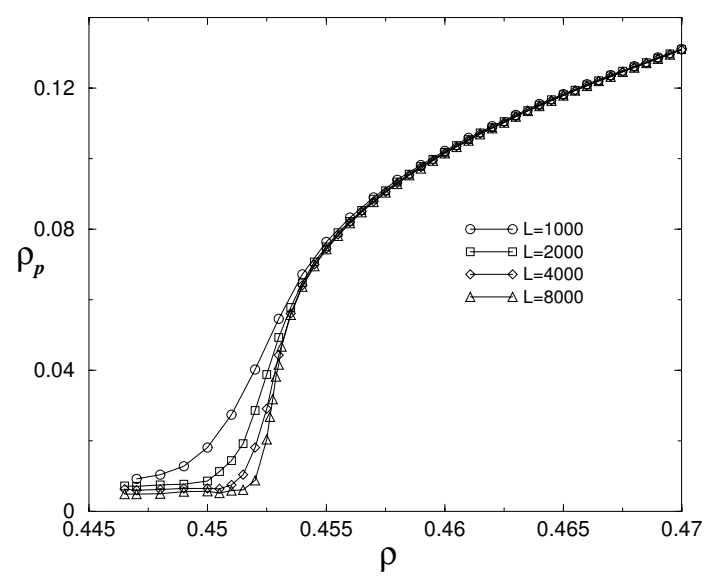

FIG. 1: Pair density $\rho_{p}$ of model 2 as a function of particle density $\rho$ for several values of the system size $L$.

$\rho_{a c}$. In actual calculation, we have used the density of pairs of adjacent particles $\rho_{p}$ defined as the average

$$
\rho_{p}=\frac{\left\langle N_{p}\right\rangle}{L},
$$

where $N_{p}$ is the total number of adjacent particles.

\section{METHODS}

To locate the critical density $\rho_{c}$ for these models, we have used three different methods. The first one consists in determining the critical density by seeking a power-law behavior of the order parameter on the system size $L$. For sufficient large systems $L$ and close to the critical point, finite-size scaling [15] establishes that the order parameter may be written in the form

$$
\rho_{p}(\Delta, L)=L^{-\beta / v} R\left(L^{1 / v} \Delta\right)
$$

where $\Delta \equiv \rho-\rho_{c}$ and the scaling function $R(x) \sim x^{\beta}$, for large $x$, since the behavior of the order parameter in this limit is given by [15]

$$
\rho_{p} \sim \Delta^{\beta}
$$

In the models studied where the tuning parameter is the density of particles, we can only change $\rho$ in increments of $1 / L$. To get around this restriction, values of $\rho_{p}$ for densities between those accessible for a given $L$ were obtained via interpolation.

A second criterion used to locate the critical point consists in determining the order parameter moment ratio $m(\rho, L)=$ $\left\langle N_{p}^{2}\right\rangle /\left\langle N_{p}\right\rangle^{2}$. According to finite size scaling, $m(\rho, L)$ obeys the scaling relation

$$
m(\rho, L)=\phi\left(L^{1 / v} \Delta\right)
$$

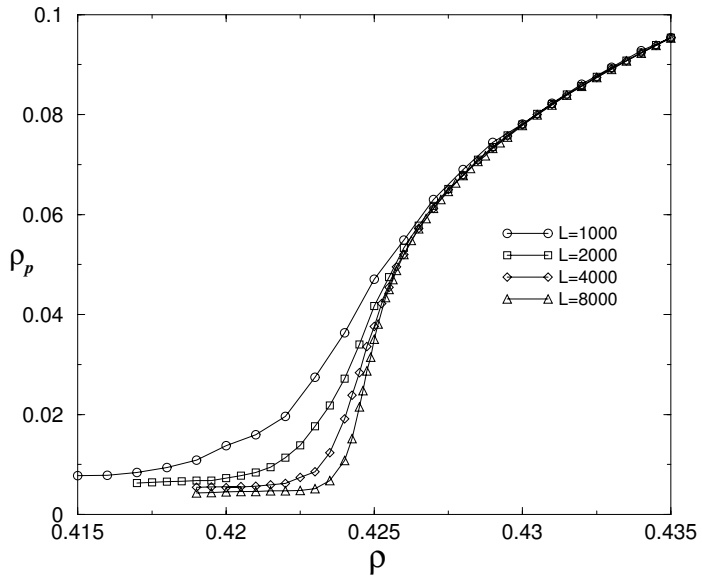

FIG. 2: Pair density $\rho_{p}$ for model 3 as a function of particle density $\rho$ for several values of the system size $L$.

where $\phi(x)$ is a universal function. This criterion is very useful to determine the transition because it is invariant with respect to the system size $L$ at the critical point.

A third criterion to locate the critical point consists in performing time-dependent simulations. This approach consists in studying the evolution of the system over a large number of independent trials (we have used from 5000 to 8000 trials), always starting with an initial configuration very close to an absorbing state with only a pair of particles. Each independent trial consists in choosing a different particle to become active and the system evolutes until a maximum time $t_{M}$. At each regular interval, we average the number of active pairs $n_{p}=\left\langle N_{p}\right\rangle$ and the survival probability $P_{s}(t)$ (defined as the probability in each time unity the system does not reach the absorbing state). For long times in the critical point these variables display power-law behavior, given by

$$
n_{p}(t) \sim t^{\eta}
$$

and

$$
P_{S}(t) \sim t^{-\delta}
$$

In off-critical regimes (supercritical and subcritical) we expect deviations from this power law behavior. Thus, this criterion is useful to locate the critical point.

\section{NUMERICAL RESULTS}

We performed extensive simulations of the models defined above. The initial condition is generated by distributing $N$ particles randomly among $L$ sites. A step of the simulation begins by randomly choosing a pair of active particles. This is done by randomly choosing a particle and its next-nearest-neighbor site. If this neighbor is occupied by a particle these two particles jump to their neighborhood according to the rules defined in section II. For the simulations, we have used systems ranging from $L=1000$ to $L=8000$ sites and $5 \times 10^{7}$ Monte Carlo 


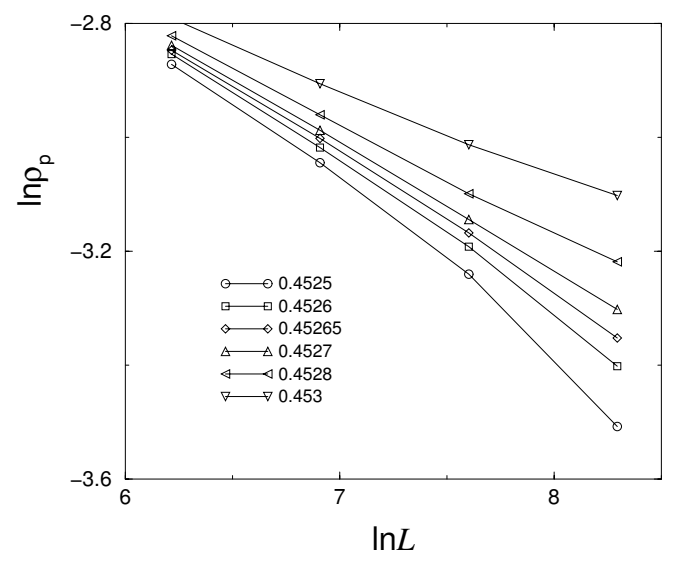

FIG. 3: Pair density $\rho_{p}$ versus system size $L$ for model 2 , for various values of the particle density.

steps. A Monte Carlo step occurs when all active sites on average are visited. For these models, we did not permit that the system fall into the absorbing state by maintaining at least an active site.

The phase transition for model 1 occurs at $\rho_{c}=1 / 2$ and was found to be discontinuous with a jump equal to 0.125 . For models 2 and 3, the transition is continuous. In Figs. 1 and 2, we show the behavior of the order parameter versus the density of particles for several values of the system size $L$. To locate the critical point we have used three methods as mentioned above. Using the cumulant method for lattices $L=4000$ and $L=8000$ the crossings occur at $\rho_{c}=0.4527$ (1) and $\rho_{c}=0.42475(5)$ for models 2 and 3 respectively. We have also performed $\log$ - $\log$ plots of $\rho_{p}$ versus $L$ for several values of $\rho$, as shown in Fig. 3 for model 2, and in Fig. 4 for model 3 , to verify the dependence of the order parameter on the lattice size. Using this criterion we find $\rho_{c}=0.45270$ (5) and $\beta / v=0.223(5)$ for model 2. For model 3, considering the critical density $\rho_{c}=0.4248$, we obtained the critical exponent $\beta / v=0.213(4)$, which agrees with the value obtained for model 2. It also agrees with the value $\beta / v=0.217(9)$ obtained by Ramasco [12].

After obtaining the critical density, we used the values of $\rho_{c}$ to calculate the critical exponents $\beta$. In Figs. 5 and 6 , we have a $\log -\log$ plot of $\rho_{p}$ versus $\Delta$ in the supercritical regime. The slope of the straight line fitted to the data point for $L=$ 8000 leads to $\beta=0.277(3)$, for model 2 , and $\beta=0.278$ (2) for model 3.

We have also performed time-dependent simulations for models 2 and 3 to determine the exponents $\delta$ and $\eta$ by means of equations (5) and (6).

$i$ From the power law behavior we found values for $\rho_{c}$ that are in agreement with the other methods. The following results are found: $\delta=0.13(1)$ and $\eta=0.36(2)$, for model 2, and $\delta=0.13(1)$ and $\eta=0.33(3)$, for model 3. To calculate the averages at each time we have used from 5000 to 8000 trials on a lattice of size $L=2000$. For comparison we have used a different way to determine the exponent $\delta$ for model

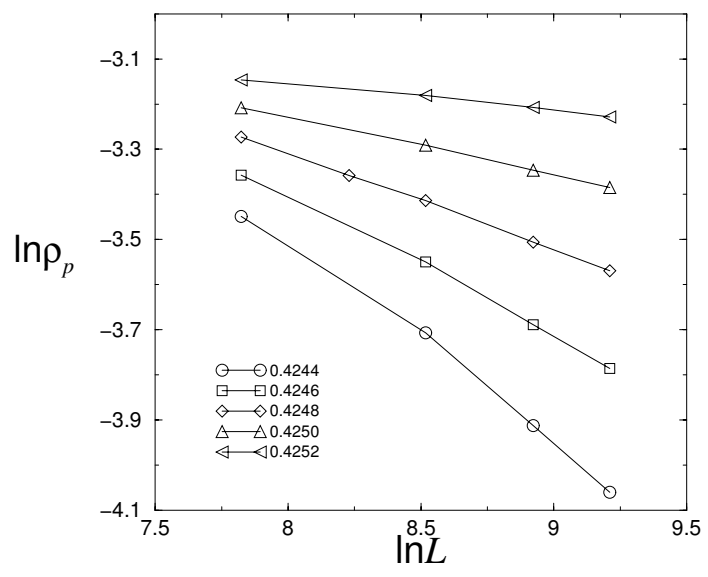

FIG. 4: Pair density $\rho_{p}$ versus system size $L$ for model 3, for various values of the particle density.

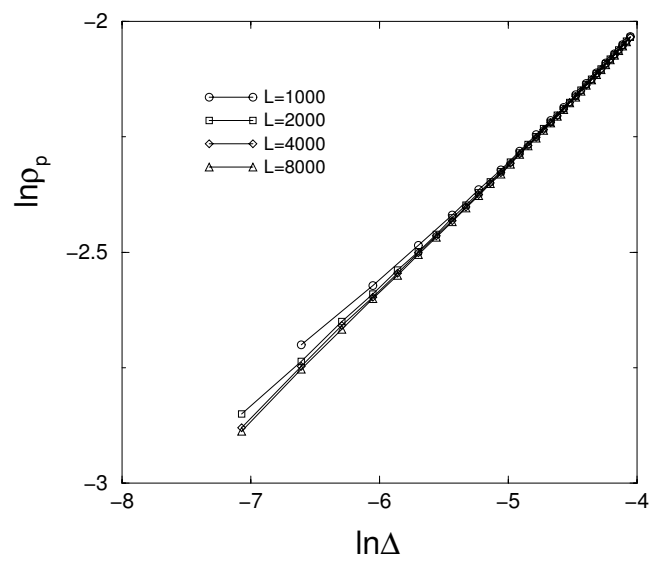

FIG. 5: Pair density $\rho_{p}$ as function of $\Delta \equiv \rho-\rho_{c}$ for values of system size $L$ ranging from 1000 to 8000 in the supercritical regime for model 2.

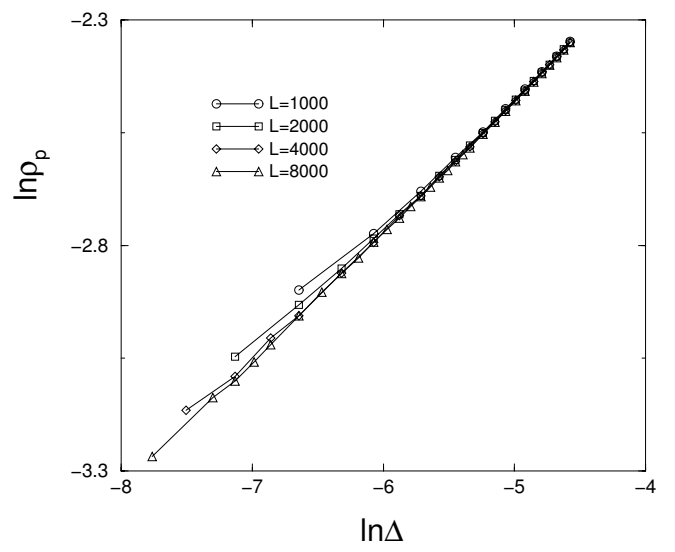

FIG. 6: Pair density $\rho_{p}$ as function of $\Delta \equiv \rho-\rho_{c}$ for values of system size $L$ ranging from 1000 to 8000 in the supercritical regime for model 3. 


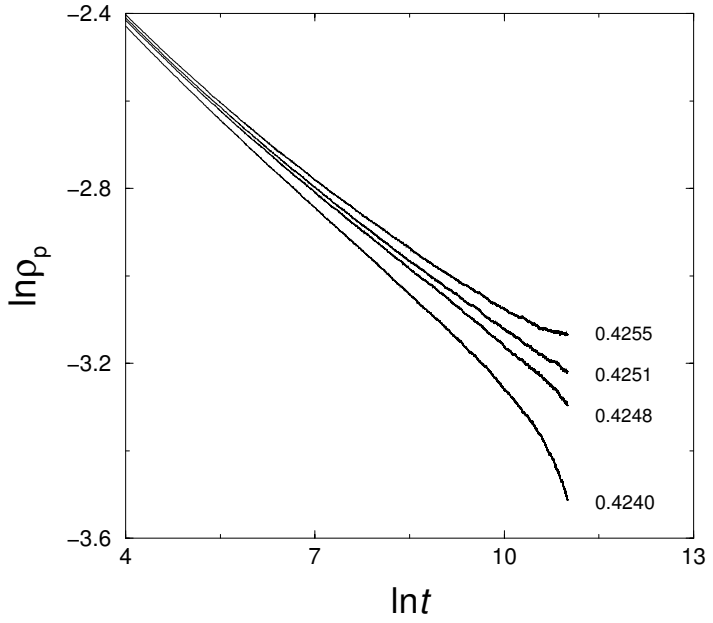

FIG. 7: Decay of the pair density $\rho_{p}$ for model 3 as function of time for several values of the particle density $\rho$ and for $L=10000$.

3. In this approach, the density of pairs $\rho_{p}$ of particles was measured as a function of time starting from a configuration obtained by distributing $N$ particles randomly. At the critical point it behaves as [15]

$$
\rho_{p} \sim t^{-\delta}
$$

The average pair density was calculated by using 5000 trials in a lattice of size $L=10000$. The data are shown in Fig. 7, from which we obtain $\delta=0.120(5)$, which is in fair agreement with the above result.

The critical exponents obtained for model 2 are presented in Table I. For comparison we also show in the same table the exponents obtained by Ramasco et al [12] and Dornic et al [13] for systems in which the order parameter is coupled to a conserved nondiffusive field. The DP universality class exponents are also shown. The result for $\beta$ is close to the DP exponent as well as to the result obtained by Ramasco et al [12]. The values of the other exponents in Table I are, however, far from the DP values. The values for $\beta / v$ and for $\eta$ are close to the results by Ramasco et al [12] and that of $\delta$ is very close to that found by Dornic et al [13].

TABLE I: Critical exponents of model 2 compared with results coming from the Langevin approach for systems with order parameter coupled to a conserved nondiffusive field $[12,13]$ and DP universality class exponents [15] in one dimension.

\begin{tabular}{|l|l|l|l|c|}
\hline$\beta$ & $\beta / \nu$ & $\delta$ & $\eta$ & Obs. \\
\hline $0.277(3)$ & $0.223(5)$ & $0.13(1)$ & $0.36(2)$ & model 2 \\
\hline $0.29(2)$ & $0.217(9)$ & $0.140(5)$ & $0.365(10)$ & {$[12]$} \\
\hline & & $0.125(2)$ & & {$[13]$} \\
\hline 0.2765 & 0.2521 & 0.1595 & 0.3137 & DP [15] \\
\hline
\end{tabular}

\section{CONCLUSION}

We have presented simple one-dimensional systems with infinitely many absorbing states and conserved particle number that possess a non trivial critical behavior. These models are modified versions of the original CLG model. In model 1 the phase transition is discontinuous. The exponents determined numerically for models 2 and 3 are in agreement with results by Ramasco et al [12] and by Dornic et al [13] for a one-dimensional system with the order parameter coupled to a conserved nondiffusive field.

\section{Acknowledgment}

C. E. F. acknowledges the financial support from Fundação de Amparo à Pesquisa do Estado de São Paulo (FAPESP) under Grant No. 03/01073-0.
[1] P. Grassberger, Z. Phys. B 47, 365 (1982).

[2] G. Grinstein, Z.-W. Lai, and D. A. Browne, Phys. Rev. A 40, 4820 (1989).

[3] M. Rossi, R. Pastor-Satorras, and A. Vespignani, Phys. Rev. Lett. 85, 1803 (2000).

[4] R. Dickman, M. A. Munõz, A. Vespignani, and S. Zaperi, Braz. J. Phys. 30, 27 (2000).

[5] A. Vespignani, R. Dickman, M. A. Munõz, and S. Zaperi, Phys. Rev. E 62, 4564 (2000).

[6] R. Dickman, T. Tomé, and M. J. de Oliveira, Phys. Rev. E 66, 016111 (2002).

[7] S. Lübeck, Phys. Rev. E 64, 016123 (2001).

[8] S. Lübeck and, Phys. Rev. E 68, 056102 (2003).

[9] S. Lübeck and P. C. Heger, Phys. Rev. E 66, 046114 (2002).
[10] R. Pastor-Satorras and A. Vespignani, Phys. Rev. E 62, R5875 (2000).

[11] A. Vespignani, R. Dickman, M. A. Muñoz, and S. Zapperi, Phys. Rev. Lett. 81, 5676 (1998).

[12] J. J. Ramasco, M. A. Munõz, and C. A. D. Santos, Phys. Rev. E 69, 045105 (2004).

[13] I. Dornic, H. Chaté, and M. Muñoz, Phys. Rev. Lett. 94, 1000601 (2005).

[14] M. J. de Oliveira, Phys. Rev. E 71, 016112 (2005).

[15] J. Marro and R. Dickman, Nonequilibrium Phase Transitions in Lattice Models (Cambridge University Press, Cambridge, 1999). 